\title{
On the Convex and Convex-Concave Solutions of Opposing Mixed Convection Boundary Layer Flow in a Porous Medium
}

\author{
M. Aïboudi $\mathbb{D}^{1}{ }^{1}$ K. Boudjema Djeffal, ${ }^{1}$ and B. Brighi ${ }^{2,3}$ \\ ${ }^{1}$ Département de Mathématiques, Faculté des Sciences Exactes et Appliquées, Université Oran 1 Ahmed Ben Bella, \\ Laboratoire de Recherche d'Analyse Mathématiques et Applications (L.A.M.A.), Oran, Algeria \\ ${ }^{2}$ Université de Haute-Alsace, LMIA EA 1108, 68100 Mulhouse, France \\ ${ }^{3}$ Université de Strasbourg, Strasbourg, France
}

Correspondence should be addressed to M. Aïboudi; m.aiboudi@yahoo.fr

Received 12 September 2018; Accepted 11 October 2018; Published 1 November 2018

Academic Editor: Paul W. Eloe

Copyright (C) 2018 M. Aïboudi et al. This is an open access article distributed under the Creative Commons Attribution License, which permits unrestricted use, distribution, and reproduction in any medium, provided the original work is properly cited.

In this paper, we are concerned with the solution of the third-order nonlinear differential equation $f^{\prime \prime \prime}+f f^{\prime \prime}+\beta f^{\prime}\left(f^{\prime}-1\right)=$ 0 , satisfying the boundary conditions $f(0)=a \in \mathbb{R}, f^{\prime}(0)=b<0$, and $f^{\prime}(t) \longrightarrow \lambda$, as $t \longrightarrow+\infty$, where $\lambda \in\{0,1\}$ and $0<\beta<1$. The problem arises in the study of the opposing mixed convection approximation in a porous medium. We prove the existence, nonexistence, and the sign of convex and convex-concave solutions of the problem above according to the mixed convection parameter $b<0$ and the temperature parameter $0<\beta<1$.

\section{Introduction}

Owing to their numerous applications in industrial manufacturing processes, the convection phenomena about heated or cooled surfaces embedded in fluid-saturated porous media have attracted considerable attention during the last few decades. In this paper, our interest focuses on the analysis of the boundary value problems $\mathscr{P}_{\lambda(a, b)}$

$$
\begin{aligned}
f^{\prime \prime \prime}+f f^{\prime \prime}+\beta f^{\prime}\left(f^{\prime}-1\right) & =0 \\
f(0) & =a, \quad a \in \mathbb{R} \\
f^{\prime}(0) & =b<0 \\
f^{\prime}(t) & \longrightarrow \lambda \text { as } t \longrightarrow+\infty
\end{aligned}
$$

where $\lambda \in\{0,1\}$. This problem derives from the study of mixed convection boundary layer near a semi-infinite vertical plate embedded in a saturated porous medium, with a prescribed power law of the distance from the leading edge for the temperature. The parameter $\beta$ is a temperature powerlaw profile and $b$ is the mixed convection parameter, namely, $b=R_{a} / P e-1$, with $R_{a}$ the Rayleigh number and $P_{e}$ the Péclet number. The interested reader can consult references $[1,2]$ for more details on the physical derivation and the numerical treatments.

Mathematical results about the problem $\mathscr{P}_{\lambda(a, b)}$ with $\lambda=$ 1 can be found in [3-7]. The case where $a \geq 0, b \geq 0, \beta>0$ and $\lambda \in\{0,1\}$ was treated by Aiboudi and al. in [3], and the results obtained generalize the ones of [6]. In [4], Brighi and Hoernel established some results about the existence and uniqueness of convex and concave solution of $\mathscr{P}_{1(a, b)}$ where $-2<\beta<0$ and $b>0$. These results can be recovered from [8], where the general equation $f^{\prime \prime \prime}+f f^{\prime \prime}+\mathbf{g}\left(f^{\prime}\right)=0$ is studied.

In [5], some theoretical results can be found about the problem $\mathscr{P}_{1(0, b)}$ with $-2<\beta<0, b=1+\varepsilon$, and $\varepsilon<$ -1 . In particular, the authors prove that there exist $\varepsilon_{*} \in$ $(-1.807,-1.806)$ and $\varepsilon^{*} \in(-1.193,-1.192)$, such that

(i) $\mathscr{P}_{1(0, b)}$ has no convex solution for any $\beta<0$ and each $\varepsilon \leq \varepsilon_{*}$.

(ii) $\mathscr{P}_{1(0, b)}$ has a convex solution for each $\beta<0$ and each $\varepsilon \in\left[\varepsilon^{*},-1\right)$.

In [7] one can find an interesting new result about the existence of convex solutions of $\mathscr{P}_{1(0, b)}$ where $0<\beta<$ 
1 under some conditions. In [5, 7], the method used by the authors allows them to prove the existence of a convex solution for the case $a=0$ and seems difficult to generalize for $a \neq 0$.

The problem $\mathscr{P}_{\lambda(a, b)}$ with $\beta=0$ is the well known Blasius problem. For a broad view, see [9]. See also [10].

Great interest is given to analytical studies of similarity solutions because of their applications in different fields, for example, in magnetohydrodynamic (see [11-13]) or in boundary layer flows (see $[8,14]$ ).

The main goal of this paper is to study the question of existence and nonexistence of the solutions of $\mathscr{P}_{\lambda(a, b)}$ with $0<$ $\beta<1$ and $\lambda \in\{0,1\}$. We will focus our attention on convex and convex-concave solutions of the equation

$$
f^{\prime \prime \prime}+f f^{\prime \prime}+\beta f^{\prime}\left(f^{\prime}-1\right)=0 \text {. }
$$

As usual, to get a convex or convex-concave solution of $\mathscr{P}_{\lambda(a, b)}$, we use the shooting technique which consists of finding the values of a parameter $c \geq 0$ for which the solution of (1) satisfying the initial conditions $f(0)=a, f^{\prime}(0)=b$, and $f^{\prime \prime}(0)=c$ exists on $[0,+\infty)$ and is such that $f^{\prime}(t) \longrightarrow \lambda$ as $t \longrightarrow+\infty$. We denote by $f_{c}$ the solution of the following initial value problem and by $\left[0, T_{c}\right.$ ) the right maximal interval of existence:

$$
\begin{aligned}
f^{\prime \prime \prime}+f f^{\prime \prime}+\beta f^{\prime}\left(f^{\prime}-1\right) & =0 \\
f(0) & =a \\
f^{\prime}(0) & =b<0 \\
f^{\prime \prime}(0) & =c \geq 0
\end{aligned}
$$

\section{On Blasius Equation}

In this section, we recall some basic properties of the subsolutions and $\varepsilon$-subsolutions of the Blasius equation. Let $I \subset \mathbb{R}$ be an interval and $f: I \longrightarrow \mathbb{R}$ be a function.

Definition 1. We say that $f$ is a subsolution of the Blasius equation $f^{\prime \prime \prime}+f f^{\prime \prime}=0$ if $f$ is of class $C^{3}$ and if $f^{\prime \prime \prime}+f f^{\prime \prime} \leq 0$ on $I$.

Definition 2 (let $\varepsilon>0$ ). We say that $f$ is an $\varepsilon$-subsolution of the Blasius equation $f^{\prime \prime \prime}+f f^{\prime \prime}=0$ if $f$ is of class $C^{3}$ and if $f^{\prime \prime \prime}+f f^{\prime \prime} \leq-\varepsilon$ on $I$.

Proposition 3 (let $t_{0} \in \mathbb{R}$ ). There does not exist nonpositive concave subsolution of the Blasius equation on the interval $\left[t_{0},+\infty\right)$.

Proof. See [8], Proposition 2.11.

Proposition 4 (let $\varepsilon>0$ and $t_{0} \in \mathbb{R}$ ). There does not exist any $\varepsilon$-subsolution of the Blasius equation on the interval $\left[t_{0},+\infty\right)$.

Proof. See [8], Proposition 2.18.

\section{Preliminary Results}

Proposition 5. Let $f$ be a solution of (1) on some maximal interval $I=\left(T_{-}, T_{+}\right)$.

(1) If $F$ is any antiderivative of $f$ on $I$, then $\left(f^{\prime \prime} e^{F}\right)^{\prime}=$ $-\beta f^{\prime}\left(f^{\prime}-1\right) e^{F}$.

(2) Assume that $T_{+}=+\infty$ and that $f^{\prime}(t) \longrightarrow \lambda \in \mathbb{R}$ as $t \longrightarrow+\infty$. If moreover $f$ is of constant sign at infinity, then $f^{\prime \prime}(t) \longrightarrow 0$ as $t \longrightarrow+\infty$.

(3) If $T_{+}=+\infty$ and if $f^{\prime}(t) \longrightarrow \lambda \in \mathbb{R}$ as $t \longrightarrow+\infty$, then $\lambda=0$ or $\lambda=1$.

(4) If $T_{+}<+\infty$, then $f^{\prime \prime}$ and $f^{\prime}$ are unbounded near $T_{+}$.

(5) If there exists a point $t_{0} \in I$ satisfying $f^{\prime \prime}\left(t_{0}\right)=0$ and $f^{\prime}\left(t_{0}\right)=\mu$, where $\mu=0$ or 1 , then, for all $t \in I$, we have $f(t)=\mu\left(t-t_{0}\right)+f\left(t_{0}\right)$.

Proof. The first item follows immediately from (1). For the proof of items (2)-(5), see [8], Proposition 3.1 with $g(x)=$ $\beta x(x-1)$.

Lemma 6. Let $\beta \in(0,1]$ and $f$ be a solution of (1) on some maximal interval $I=\left(T_{-}, T_{+}\right)$. If there exists $t_{0} \in I$ such that

$$
\begin{aligned}
f^{\prime}\left(t_{0}\right) & >1 \text { and } \\
f\left(t_{0}\right)\left(1-f^{\prime}\left(t_{0}\right)\right) & \leq f^{\prime \prime}\left(t_{0}\right) \leq 0,
\end{aligned}
$$

then $T_{+}=+\infty$ and $f^{\prime}(t) \longrightarrow 1$ as $t \longrightarrow+\infty$. Moreover, $f^{\prime \prime}<$ 0 on $\left[t_{0},+\infty\right)$.

Proof. See [3], Lemma 9.

\section{The Boundary Value Problem in the Convex and Convex-Concave Case with $0<\beta<1$}

In the following, we take $a, b \in \mathbb{R}$ and $\lambda \in\{0,1\}$ with $b<0$ and $0<\beta<1$. We are interested here in convex and convexconcave solutions of the boundary value problem $\mathscr{P}_{\lambda(a, b)}$. As mentioned in the introduction, we will use the shooting method to find these solutions. Define the following sets:

$$
\begin{aligned}
C_{1} & =\left\{c \geq 0: f_{c}^{\prime} \leq 0 \text { and } f_{c}^{\prime \prime} \geq 0 \text { on }\left[0, T_{c}\right)\right\}, \\
C_{2} & =\left\{c \geq 0: \exists t_{c} \in\left[0, T_{c}\right), \exists \varepsilon_{c}>0 \text { s.t } f_{c}^{\prime}\right. \\
& <0 \text { on }\left(0, t_{c}\right), f_{c}^{\prime}>0 \text { on }\left(t_{c}, t_{c}+\varepsilon_{c}\right) \text { and } f_{c}^{\prime \prime} \\
& \left.>0 \text { on }\left(0, t_{c}+\varepsilon_{c}\right)\right\}, \\
C_{3} & =\left\{c \geq 0: \exists s_{c} \in\left[0, T_{c}\right), \exists \varepsilon_{c}>0 \text { s.t } f_{c}^{\prime \prime}\right. \\
& >0 \text { on }\left(0, s_{c}\right), f_{c}^{\prime \prime}<0 \text { on }\left(s_{c}, s_{c}+\varepsilon_{c}\right) \text { and } f_{c}^{\prime} \\
& \left.<0 \text { on }\left(0, s_{c}+\varepsilon_{c}\right)\right\} .
\end{aligned}
$$

Remark 7. It is easy to prove that $C_{2}$ and $C_{3}$ are disjoint nonempty open subsets of $[0,+\infty)$ and that there exist $c_{0}>$ 
$c_{*}>0$ such that $C_{2}=\left(c_{0},+\infty\right), C_{3}=\left[0, c_{*}\right)$, and $C_{1} \cup C_{2} \cup$ $C_{3}=[0,+\infty)$ (see Appendix A of [8] with $g(x)=\beta x(x-1)$ and $\beta>0)$.

Lemma 8 (let $\beta>0$ ). Then, $f_{c}$ is a convex solution of the boundary value problem $\mathscr{P}_{0(a, b)}$ if and only if $c \in C_{1}$.

Proof. See Appendix A of [8] with $g(x)=\beta x(x-1)$ and $\beta>$ 0 .

Lemma 9 (let $\beta>0$ ). If $c \in C_{3}$, then $T_{c}<+\infty$. Moreover, $f_{c}$ is convex-concave, decreasing and $f_{c}^{\prime}(t) \longrightarrow-\infty$ as $t \longrightarrow T_{c}$.

Proof. If $c \in C_{3}$ then there exists $s_{c} \in\left[0, T_{c}\right)$ such that $f_{c}^{\prime}\left(s_{c}\right)<$ 0 and $f_{c}^{\prime \prime}\left(s_{c}\right)=0$. From Proposition 5, items (1) and (3), we have $f_{c}^{\prime \prime}(t)<0$ and $f_{c}^{\prime}(t)<0$ for all $t \in\left(s_{c}, T_{c}\right)$, and $f_{c}^{\prime}(T) \longrightarrow$ $-\infty$ as $t \longrightarrow T_{c}$. Thus, $f_{c}$ is convex-concave solution on $\left[0, T_{c}\right)$ and $f_{c}^{\prime}(t) \longrightarrow-\infty$ as $t \longrightarrow T_{c}$.

Let us assume that $T_{c}=+\infty$; then there exists $t_{0} \in$ $\left(s_{c},+\infty\right)$ such that $f_{c}^{\prime}$ and $f_{c}$ are negative on $t \in\left(t_{0},+\infty\right)$ and we obtain $f_{c}^{\prime \prime \prime}+f_{c} f_{c}^{\prime \prime}=-\beta f_{c}^{\prime}\left(f_{c}^{\prime}-1\right)<0$ on $\left(t_{0},+\infty\right)$. Hence, $f_{c}$ is a nonpositive concave subsolution of the Blasius equation on $\left(t_{0},+\infty\right)$. This contradicts the Proposition 3 and thus $T_{c}<+\infty$.

Remark 10. From Proposition 5, items (1), (3), and (5), if $c \in$ $C_{2}$, then there are only three possibilities for the solution of the initial value problem $\mathscr{P}_{(a, b, c)}$ :

(1) $f_{c}$ is convex and $f_{c}^{\prime}(t) \longrightarrow+\infty$ as $t \longrightarrow T_{c}$ (with $T_{c} \leq$ $+\infty)$.

(2) There exists a point $t_{0} \in\left[0, T_{c}\right)$ such that $f_{c}^{\prime \prime}\left(t_{0}\right)=0$ and $f_{c}^{\prime}\left(t_{0}\right)>1$.

(3) $f_{c}$ is a convex solution of $\mathscr{P}_{1(a, b)}$.

The next proposition shows that case (1) cannot hold.

Proposition 11 (let $\beta>0$ ). There does not exist $c \geq 0$, such that $f_{c}$ is convex on its right maximal interval of existence $\left[0, T_{c}\right)$ and $f_{c}^{\prime}(t) \longrightarrow+\infty$ as $t \longrightarrow T_{c}$.

Proof. Assume that $f_{c}$ is convex on its right maximal interval of existence $\left[0, T_{c}\right)$ and $f_{c}^{\prime}(t) \longrightarrow+\infty$ as $t \longrightarrow T_{c}$. Then there exists $t_{0} \in\left[0, T_{c}\right)$ such that, for all $t \in\left[t_{0}, T_{c}\right), f_{c}^{\prime}(t)>1$ and

$$
\begin{aligned}
f_{c}^{\prime \prime \prime}(t)+f_{c}(t) f_{c}^{\prime \prime}(t) & =-\beta f_{c}^{\prime}(t)\left(f_{c}^{\prime}(t)-1\right) \\
& <-\beta f_{c}^{\prime}\left(t_{0}\right)\left(f_{c}^{\prime}\left(t_{0}\right)-1\right)=-\varepsilon .
\end{aligned}
$$

Consequently, $f_{c}$ is a $\varepsilon$-subsolution of the Blasius equation on $\left[t_{0}, T_{c}\right)$. Therefore from Proposition 4 we have $T_{c}<+\infty$.

Furthermore, there exists $t_{1} \in\left[t_{0}, T_{c}\right)$ such that $f_{c}\left(t_{1}\right)=$ $\alpha>0$ and $f_{c}^{\prime}\left(t_{1}\right)>1$, and then $f_{c}^{\prime \prime \prime}(t)+f_{c}(t) f_{c}^{\prime \prime}(t)<0$ and $f_{c}(t)>f_{c}\left(t_{1}\right)=\alpha$ for all $t \in\left[t_{1}, T_{c}\right)$. Thus,

$$
f_{c}^{\prime \prime \prime}(t)<-\alpha f_{c}^{\prime \prime}(t)
$$

for all $t \in\left[t_{1}, T_{c}\right)$. Next, integrating (5) on $\left[t_{1}, t\right]$ for $t_{1}<t<$ $T_{c}$, we obtain $f_{c}^{\prime \prime}(t)-f_{c}^{\prime \prime}\left(t_{1}\right)<-\alpha\left(f_{c}^{\prime}(t)-f_{c}^{\prime}\left(t_{1}\right)\right)$ and using Proposition 5, item (4), yields a contradiction as $t \longrightarrow T_{c}$.

\section{The $a \leq 0$ Case}

Lemma 12 (let $0<\beta<1$ and $a \leq 0$ ). If $c \geq 0$ and if there exists $t_{0} \in\left[0, T_{c}\right)$ such that $f_{c}^{\prime \prime}\left(t_{0}\right)=0$ and $f_{c}^{\prime}\left(t_{0}\right)>1$, then $f_{c}\left(t_{0}\right)>0$.

Proof. Let $c \geq 0$ and assume that there exists $t_{0} \in\left[0, T_{c}\right)$ such that $f_{c}^{\prime \prime}\left(t_{0}\right)=0$ and $f_{c}^{\prime}\left(t_{0}\right)>1$.

Let us consider the function $H_{c}=f_{c}^{\prime \prime}+f_{c}\left(f_{c}^{\prime}-\beta\right)$. Since $H_{c}^{\prime}=(1-\beta) f_{c}^{\prime 2} \geq 0$ on $\left[0, T_{c}\right)$, then $H_{c}$ is nondecreasing on $\left[0, T_{c}\right)$ and hence

$$
\begin{aligned}
0 & \leq H_{c}(0)=c+a(b-\beta)<H_{c}\left(t_{0}\right) \\
& =f_{c}\left(t_{0}\right)\left(f_{c}^{\prime}\left(t_{0}\right)-\beta\right) .
\end{aligned}
$$

Thus, $f_{c}\left(t_{0}\right)>0$.

For the rest of this section we will set $a^{*}=$ $-\sqrt{\left(1-b^{2}\right) /(\beta-2 b)}$.

Proposition 13 (let $0<\beta<1$ ). If either $b \leq-1$ or $b \in(-1,0$ ] and $a \leq a^{*}$, then the boundary value problem $\mathscr{P}_{1(a, b)}$ has no convex solution.

Proof. Suppose that $b \leq-1$ and that $f_{c}$ is a convex solution of the boundary value problem $\mathscr{P}_{1(a, b)}$. Then, there exists $t_{*}>0$ such that $f_{c}\left(t_{*}\right)=0$.

Let $K_{c}=2 f_{c} f_{c}^{\prime \prime}-f_{c}^{\prime 2}+f_{c}^{2}\left(2 f_{c}^{\prime}-\beta\right)$. From (1), we obtain $K_{c}^{\prime}=2(2-\beta) f_{c} f_{c}^{\prime 2}<0$ on $\left(0, t_{*}\right)$. Therefore, $K_{c}$ is decreasing on $\left(0, t_{*}\right)$ and hence $K_{c}(0)>K_{c}\left(t_{*}\right)$. It follows that

$$
f_{c}^{\prime 2}\left(t_{*}\right)>-2 a c+b^{2}+a^{2}(\beta-2 b) \geq b^{2},
$$

which implies that $f_{c}^{\prime}\left(t_{*}\right)>1$. This is a contradiction. The same contradiction is obtained where $b \in(-1,0]$ and $a \leq$ $a^{*}$.

Theorem 14. Let $0<\beta<1$ and $a, b \in \mathbb{R}$ with $b<0$ and $a \leq 0$ and $0<\beta<1$.

(1) The boundary value problem $\mathscr{P}_{0(a, b)}$ has at least one convex solution.

(2) If either $b \leq-1$ or $b \in(-1,0]$ and $a \leq a^{*}$, then the boundary value problem $\mathscr{P}_{1(a, b)}$ has no convex solution and has infinitely many convex-concave solutions.

Proof. The first result follows from Remark 7 and Lemma 8. The second result follows from Remark 7, Remark 10, Proposition 11, Proposition 13, and Lemma 6.

\section{The $a>0$ Case}

Let $a, b \in \mathbb{R}$ with $b<0$ and $a>0$. We assume $0<\beta<1$ and consider the solution $f_{c}$ of the initial value problem $P_{(a, b, c)}$ on the right maximal interval of existence $\left[0, T_{c}\right)$.

Let us set $b^{*}=\max \left\{-(1 / 2) a^{2},-\beta /(1-\beta)\right\}$.

Lemma 15 (let $0<\beta<1$. let $c \geq 0$ ). If $b \in\left(b^{*}, 0\right)$ and if there exists $t_{*} \in\left(0, T_{c}\right)$ such that $t_{*}$ is the first point where $f_{c}\left(t_{*}\right)=0$, then $f_{c}^{\prime}\left(t_{*}\right)<0$ and $f_{c}^{\prime \prime}\left(t_{*}\right)<0$. 
Proof. Let $t_{*} \in\left(0, T_{c}\right)$ be such that $f_{c}>0$ on $\left[0, t_{*}\right)$ and $f_{c}\left(t_{*}\right)=0$. Suppose that $f_{c}^{\prime \prime}>0$ on $\left[0, t_{*}\right)$. Then, necessarily, we have $f_{c}^{\prime}<0$ on $\left[0, t_{*}\right)$. Moreover, since $f_{c}^{\prime}$ is increasing and $b>b^{*}$, we also have $f_{c}^{\prime}>-\beta /(1-\beta)$ on $\left[0, t_{*}\right)$.

Let $E_{c}=f_{c}^{\prime \prime}+f_{c} f_{c}^{\prime}$. From $(1)$, we have $E_{c}^{\prime}=(1-\beta) f_{c}^{\prime 2}+\beta f_{c}^{\prime}$. Consequently, $E_{c}^{\prime}<0$ on $\left[0, t_{*}\right)$ and since $E_{c}\left(t_{*}\right)=f_{c}^{\prime \prime}\left(t_{*}\right) \geq$ 0 , it follows that $E_{c}>0$ on $\left[0, t_{*}\right)$. Integrating from 0 to $t_{*}$ gives

$$
0<\int_{0}^{t_{*}} E_{c}(t) \mathrm{d} t=f_{c}^{\prime}\left(t_{*}\right)-b-\frac{1}{2} a^{2} .
$$

Thus $f_{c}^{\prime}\left(t_{*}\right)>b+(1 / 2) a^{2} \geq 0$ which is a contradiction.

Therefore, there exists $t_{0} \in\left[0, t_{*}\right)$ such that $f_{c}^{\prime \prime}>0$ on $\left(0, t_{0}\right)$ and $f_{c}^{\prime \prime}\left(t_{0}\right)=0$. From Proposition 5, items (1) and (5), we have either $f_{c}^{\prime}\left(t_{0}\right)<0$ or $f_{c}^{\prime}\left(t_{0}\right)>1$. The second case cannot happen. Assume, for the sake of contradiction, that $f_{c}^{\prime}\left(t_{0}\right)>1$. Then $f_{c}^{\prime \prime} \geq 0$ and $f_{c}>0$ on $\left[0, t_{0}\right]$, so that we have $f_{c}\left(t_{0}\right)\left(1-f_{c}^{\prime}\left(t_{0}\right)\right) \leq f_{c}^{\prime \prime}\left(t_{0}\right) \leq 0$. From Lemma 6 , we obtain that $T_{c}=+\infty, f_{c}^{\prime}(t) \longrightarrow 1$ as $t \longrightarrow+\infty$, and $f_{c}^{\prime \prime}<0$ on $\left[t_{0},+\infty\right)$. It follows that $f_{c}$ is a positive convex-concave solution of the boundary value problem $\mathscr{P}_{1(a, b)}$ on $[0,+\infty)$, which contradicts the existence of $t_{*}$. Consequently, we have $f_{c}^{\prime}\left(t_{0}\right)<0$. This implies that $f_{c}^{\prime}<0$ on $\left[0, t_{0}\right]$ and that $c \in C_{3}$. By virtue of Lemma 9 , we see that $f_{c}^{\prime \prime}$ remains negative after $t_{0}$. The proof is complete.

Lemma 16 (let $0<\beta<1$ ). If there exists $t_{0} \in\left[0, T_{c}\right)$ such that $f_{c}^{\prime \prime}\left(t_{0}\right)=0$ and $f_{c}^{\prime}\left(t_{0}\right)>1$, then $f_{c}\left(t_{0}\right)>0$.

Proof. Assume that there exists $t_{0} \in\left[0, T_{c}\right)$ such that $f_{c}^{\prime \prime}\left(t_{0}\right)=$ $0, f_{c}^{\prime}\left(t_{0}\right)>1$, and $f_{c}\left(t_{0}\right)<0$. Then, there would exist $t_{1}<t_{0}$ such that $f_{c}\left(t_{1}\right)=0$.

Let $H_{c}=f_{c}^{\prime \prime}+f_{c}\left(f_{c}^{\prime}-\beta\right)$. From (1), we have $H_{c}^{\prime}=$ $(1-\beta) f_{c}^{\prime 2} \geq 0$ on $\left[0, T_{c}\right)$. Therefore, $H_{c}$ is nondecreasing on $\left[0, T_{c}\right)$. Since $H_{c}\left(t_{0}\right)=f_{c}\left(t_{0}\right)\left(f_{c}^{\prime}\left(t_{0}\right)-\beta\right)<0$, we get $f_{c}^{\prime \prime}\left(t_{1}\right)=H_{c}\left(t_{1}\right)<0$. But, this and Proposition 5, item (1), imply that $f_{c}^{\prime \prime}$ remains negative on $\left(t_{1}, T_{c}\right)$, a contradiction. Hence $f_{c}\left(t_{0}\right)>0$.

Lemma 17. If $0<\beta<1$ and $b \in\left(b^{*}, 0\right)$, then there exists $c_{0} \in C_{2}$ such that if $c \geq c_{0}$ then $f_{c}$ is a convex-concave solution of $\mathscr{P}_{1(a, b)}$.

Proof [let $c \in C_{2}$ ]. From Remark 10 and Proposition 11, we see that either $f_{c}$ is a convex solution of $\mathscr{P}_{1(a, b)}$ or there exists $t_{0} \in\left[0, T_{c}\right)$ such that $f_{c}^{\prime \prime}\left(t_{0}\right)=0$ and $f_{c}^{\prime}\left(t_{0}\right)>1$. Now, as we have seen in the proof of Lemma 15 , in the second case, $f_{c}$ is a convex-concave solution of $\mathscr{P}_{1(a, b)}$.

Let $c \in C_{2}$ be such that $f_{c}$ is a convex solution of $\mathscr{P}_{1(a, b)}$. Therefore, we have $b<f_{c}^{\prime}<1$ on $[0,+\infty)$ and, from Lemma 15 , we have $f_{c}>0$. It follows that

$$
\begin{aligned}
\left(f_{c}^{\prime \prime}+f_{c}\left(f_{c}^{\prime}-1\right)\right)^{\prime} & =(1-\beta) f_{c}^{\prime}\left(f_{c}^{\prime}-1\right) \\
& \geq-\frac{1}{4}(1-\beta)
\end{aligned}
$$

on $[0,+\infty)$. Integrating between 0 and $t \geq 0$, and using the fact that $f_{c}>0$, we obtain

$$
\begin{aligned}
f_{c}^{\prime \prime}(t) \geq & -\frac{1}{4}(1-\beta) t+a(b-1)+c \\
& -f_{c}(t)\left(f_{c}^{\prime}(t)-1\right) \\
\geq & -\frac{1}{4}(1-\beta) t+a(b-1)+c .
\end{aligned}
$$

Integrating once again we get

$$
\begin{aligned}
& \forall t \geq 0 \\
& 1>f_{c}^{\prime}(t) \geq-\frac{1}{8}(1-\beta) t^{2}+(a(b-1)+c) t+b .
\end{aligned}
$$

Let us set $P_{c}(t)=-(1 / 8)(1-\beta) t^{2}+(a(b-1)+c) t+b-1$. We have $P_{c}(t)<0$ for all $t \geq 0$. It means that $P_{c}$ has no positive roots. Thus $c$ cannot be too large, because, on the contrary, its discriminant $\Delta=(a(b-1)+c)^{2}+(1 / 2)(1-\beta)(b-1)$ and $a(b-1)+c$ would be positive, and hence the polynomial $P_{c}$ would have two positive roots, a contradiction.

Therefore, there exists $c_{0}>0$ such that $f_{c}$ is convexconcave solution of the problem $\mathscr{P}_{1(a, b)}$ for $c \geq c_{0}$. This completes the proof.

Theorem 18. Let $a, b \in \mathbb{R}$, with $b<0, a>0$, and $0<\beta<1$.

(1) The boundary value problem $\mathscr{P}_{0(a, b)}$ has at least one convex solution. If in addition $b \in\left(b^{*}, 0\right)$, then any convex solution of $\mathscr{P}_{0(a, b)}$ is positive.

(2) If $b \in\left(b^{*}, 0\right)$, then the boundary value problem $\mathscr{P}_{1(a, b)}$ has infinitely many positive convex-concave solutions.

Proof. The first part of (1) follows from Remark 7 and Lemma 8. The second part follows from Lemma 15, because if there was a point $t_{*}$ such that $f_{c}>0$ on $\left[0, t_{*}\right)$ and $f_{c}\left(t_{*}\right)=0$ then $f_{c}^{\prime \prime}\left(t_{*}\right)<0$, a contradiction. The second result follows from Remark 7, Remark 10, Proposition 11, Lemma 16, and Lemma 6.

\section{Conclusion}

In this work, in particular in Theorems 14 and 18, we have presented some new and important results about the boundary value problems $\mathscr{P}_{0(a, b)}$ and $\mathscr{P}_{1(a, b)}$, which we summarize below. The parameters $\beta$ and $b$ satisfy $0<\beta<1$ and $b<0$. The constants $a_{*}$ and $b_{*}$ are defined in Sections 5 and 6 .

(1) For $a \leq 0$ :

(a) The boundary value problem $\mathscr{P}_{0(a, b)}$ has at least one convex solution.

(b) If either $b \leq-1$ or $b \in(-1,0]$ and $a \leq a^{*}$, then the boundary value problem $\mathscr{P}_{1(a, b)}$ has no convex solution and has infinitely many convexconcave solutions.

(2) For $a>0$ : 
(a) If $b \in\left(b^{*}, 0\right)$, then the boundary value problem $\mathscr{P}_{0(a, b)}$ has at least one positive convex solution.

(b) If $b \in\left(b^{*}, 0\right)$, then the boundary value problem $\mathscr{P}_{1(a, b)}$ has infinitely many positive convexconcave solutions.

Numerical simulations prompt us to formulate the following conjecture.

Conjecture 19. Let $a, b \in \mathbb{R}$, with $b \leq-1, a>0$, and $0<\beta<$ 1. The boundary value problem $\mathscr{P}_{1(a, b)}$ has no convex solution.

To finish, we give the following proposition concerning the case $a=0$.

Proposition 20 (let $\beta<2$ ). If $b \leq-1$, then the boundary value problem $\mathscr{P}_{1(0, b)}$ has no convex solution.

Proof. Assume that $f_{c}$ is a convex solution of the boundary value problem $\mathscr{P}_{1(0, b)}$. Then, there exists $t_{*} \geq 0$, such that $f_{c}<0$ on $\left(0, t_{*}\right), f_{c}\left(t_{*}\right)=0$, and $f_{c}^{\prime}\left(t_{*}\right)>0$. Consider again the function

$$
K_{c}=2 f_{c} f_{c}^{\prime \prime}-f_{c}^{\prime 2}+f_{c}^{2}\left(2 f_{c}^{\prime}-\beta\right) .
$$

We have $K_{c}^{\prime}=2(2-\beta) f_{c} f_{c}^{\prime 2}<0$ on $\left(0, t_{*}\right)$. Thus, $K_{c}$ is a decreasing function and hence $K_{c}(0)>K_{c}\left(t_{*}\right)$. It follows that $f_{c}^{\prime 2}\left(t_{*}\right)>b^{2}$ which implies that $f_{c}^{\prime}\left(t_{*}\right)>1$, which is a contradiction.

\section{Data Availability}

The data used to support the findings of this study are available from the corresponding author upon request.

\section{Conflicts of Interest}

The authors declare that they have no conflicts of interest.

\section{References}

[1] E. H. Aly, L. Elliott, and D. B. Ingham, "Mixed convection boundary-layer flow over a vertical surface embedded in a porous medium," European Journal of Mechanics - B/Fluids, vol. 22, no. 6, pp. 529-543, 2003.

[2] R. Nazar, N. Amin, and I. Pop, "Unsteady mixed convection boundary layer flow near the stagnation point on a vertical surface in a porous medium," International Journal of Heat and Mass Transfer, vol. 47, no. 12-13, pp. 2681-2688, 2004.

[3] M. Aiboudi, I. Bensari-Khelil, and B. Brighi, "Similarity solutions of mixed convection boundary-layer flows in a porous medium," Differential Equations \& Applications, vol. 9, no. 1, pp. 69-85, 2017.

[4] B. Brighi and J.-D. Hoernel, "On the concave and convex solutions of a mixed convection boundary layer approximation in a porous medium," Applied Mathematics Letters, vol. 19, no. 1, pp. 69-74, 2006.

[5] G. C. Yang, L. Zhang, and L. F. Dang, "Existence and nonexistence of solutions on opposing mixed convection problems in boundary layer theory," European Journal of Mechanics B/Fluids, vol. 43, pp. 148-153, 2014.
[6] M. Guedda, "Multiple solutions of mixed convection boundarylayer approximations in a porous medium," Applied Mathematics Letters, vol. 19, no. 1, pp. 63-68, 2006.

[7] G. C. Yang, "An extension result of the opposing mixed convection problem arising in boundary layer theory," Applied Mathematics Letters, vol. 38, pp. 180-185, 2014.

[8] B. Brighi, "The equation $\mathrm{f}^{\prime \prime}+\mathrm{ff} "+\mathrm{g}(\mathrm{f})=0$ and the associated boundary value problems," Results in Mathematics, vol. 61, no. 3-4, pp. 355-391, 2012.

[9] B. Brighi, A. Fruchard, and T. Sari, "On the Blasius problem," Advances in Differential Equations, vol. 13, no. 5-6, pp. 509-600, 2008.

[10] G. C. Yang, "An upper bound on the critical value $\beta^{\star}$ involved in the Blasius problem," Journal of Inequalities and Applications, Article ID 960365, 6 pages, 2010.

[11] F. A. Hendi and M. Hussain, "Analytic solution for MHD Falkner-Skan flow over a porous surface," Journal of Applied Mathematics, vol. 2012, 2012.

[12] M. Guedda and A. Ouahsine, "Similarity solutions of MHD flows in a saturated porous medium," European Journal of Mechanics - B/Fluids, vol. 33, pp. 87-94, 2012.

[13] O. D. Makinde and A. Aziz, "MHD mixed convection from a vertical plate embedded in a porous medium with a convective boundary condition," International Journal of Thermal Sciences, vol. 49, no. 9, pp. 1813-1820, 2010.

[14] M. Guedda, E. H. Aly, and A. Ouahsine, "Analytical and ChPDM analysis of MHD mixed convection over a vertical flat plate embedded in a porous medium filled with water at $4^{\circ} \mathrm{C}$," Applied Mathematical Modelling, vol. 35, no. 10, pp. 5182-5197, 2011. 


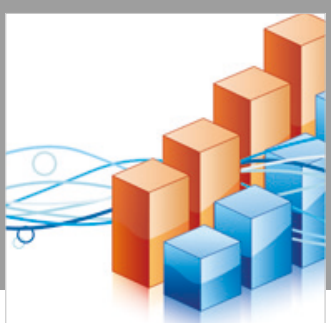

Advances in

Operations Research

\section{-n-m}
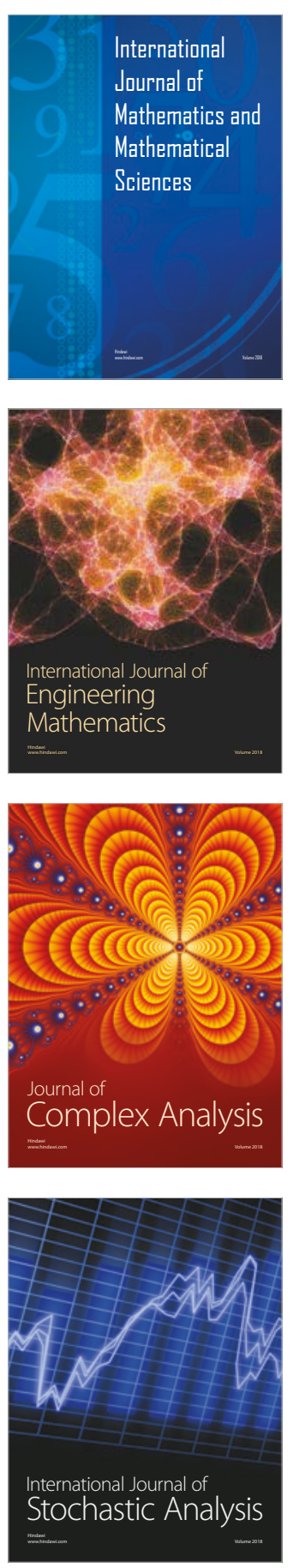
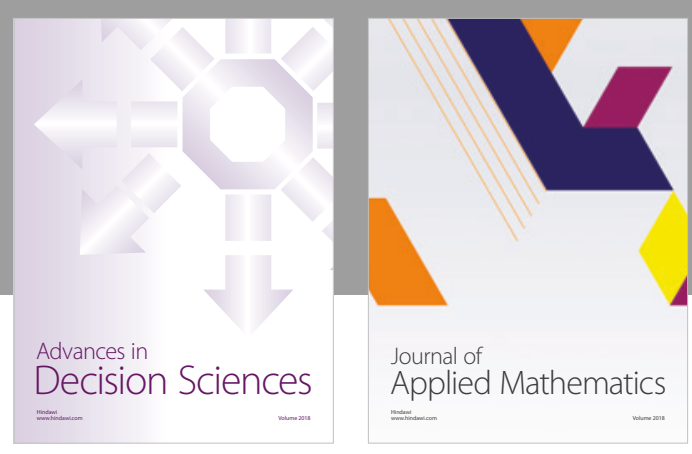

Journal of

Applied Mathematics
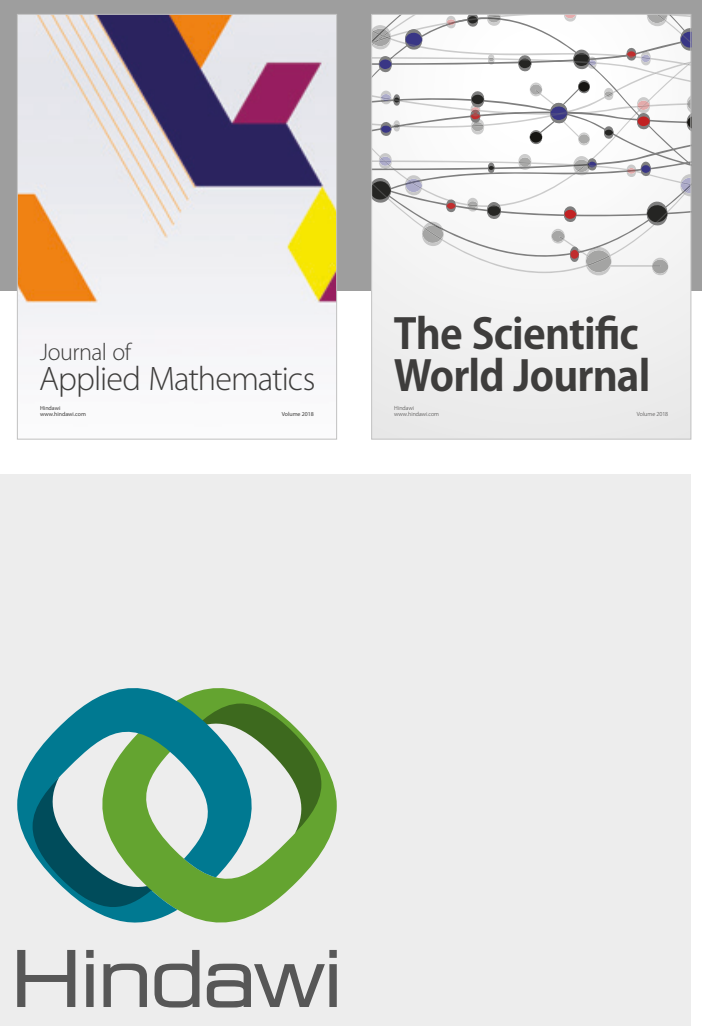

Submit your manuscripts at

www.hindawi.com

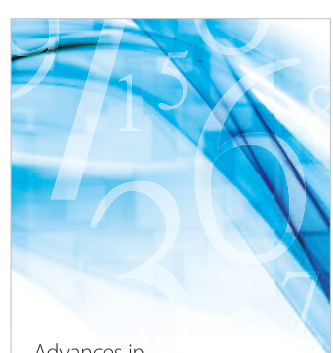

Advances in
Numerical Analysis
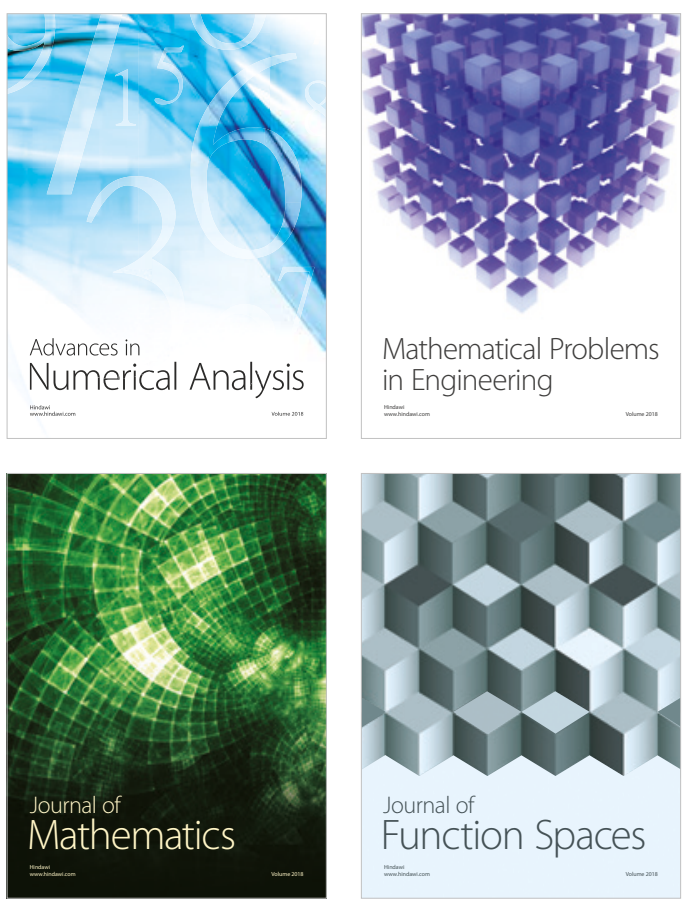

Mathematical Problems in Engineering

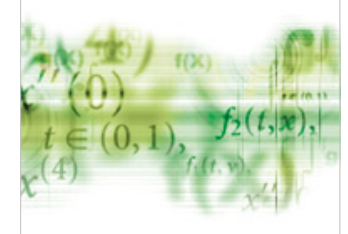

International Journal of

Differential Equations

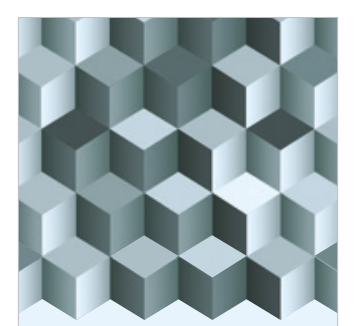

Journal of

Function Spaces

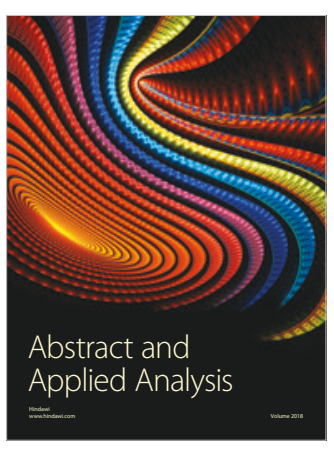

The Scientific

World Journal

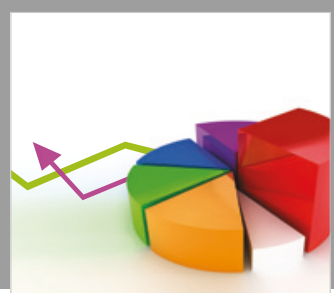

Journal of

Probability and Statistics
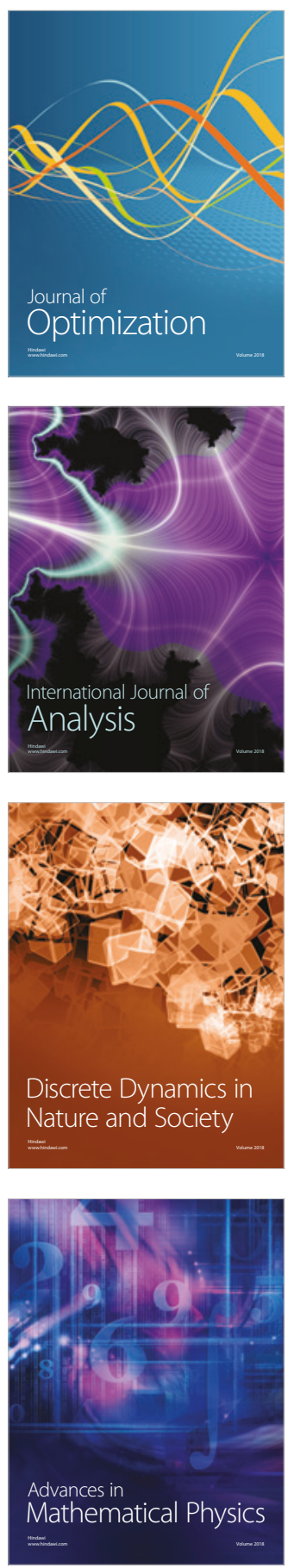